\title{
Estilo emocional, salud y calidad de vida percibida en embarazadas
}

\section{Emotional style, health and perceived quality of life in pregnants}

\author{
Leticia Guarino $^{1 *}$, Fausto Scremín** y Sonia Borrás* \\ * Dpto. de Ciencia y Tecnología del Comportamiento \\ Universidad Simón Bolívar, \\ Caracas, Venezuela \\ ** Instituto Venezolano de los Seguros Sociales \\ Caracas, Venezuela
}

(Recepción: Enero 2010 - Aceptación: Junio 2010)

\begin{abstract}
Resumen
El presente estudio tiene como objetivo determinar las posibles relaciones entre el estilo emocional (rumiación e inhibición emocional) e indicadores de salud y calidad de vida percibida en mujeres embarazadas. Para ello, un grupo de 94 mujeres embarazadas venezolanas en el primer trimestre de gestación completaron cuestionarios que miden las variables estudiadas, a saber: Rumiación, Inhibición Emocional, Salud global y Calidad de Vida percibida. Los resultados apoyan hallazgos previos sobre la asociación positiva que existe entre un estilo emocional negativo y un mayor deterioro en la salud, así como aporta nuevas evidencias sobre la relación inversa que existe entre esta diferencia individual y la calidad de vida, en este caso en un grupo poco estudiado hasta el momento en su dimensión psicosocial como lo son las mujeres embarazadas.
\end{abstract}

Palabras claves: estilo emocional, salud, calidad de vida, embarazo.

\begin{abstract}
The aim of the present study is to determine the possible relationship among emotional style (rumination and emotional inhibition) and the perceived health and quality of life of pregnant women. To do so, a sample of 94 Venezuelan women on their first trimester of pregnancy completed questionnaires measuring the studied variables: Rumination, Emotional Inhibition, Global Health and perceived Quality of Life. Results support previous findings regarding the positive association between negative emotional style and the deterioration of the health status, while brings new evidence of the inverse relationship between these individual difference and the quality of life in this particular group, who has been poorly studied in its psychosocial dimension.
\end{abstract}

Key words: emotional style, health, quality of life, pregnancy.

1 Correspondencia a Leticia Guarino. Valle de Sartenejas, Aptdo. Postal 89.000 - A. Edo. Miranda, Venezuela. Teléfono: 00 58212 9063595. Fax: 0058212906 3811. E-mail: lguarino@usb.ve. 


\section{Introducción}

La comprensión cabal del concepto de calidad de vida, requiere de la inclusión tanto de los aspectos objetivos (salud, alimentación, educación, trabajo, vivienda, seguridad social, vestidos y derechos humanos) como de los subjetivos (percepción de bienestar). En este sentido, Levi y Anderson (1980) la definen como una medida compuesta de bienestar físico, mental y social, tal y como lo percibe cada individuo y cada grupo. Por su parte, Shin y Johnson (1978) proponen una definición que puede ser operativizada: la posesión de los recursos necesarios para la satisfacción de las necesidades y deseos individuales, la participación en las actividades que permitan el desarrollo personal y la comparación satisfactoria con los demás, aspectos todos que dependen del conocimiento y la experiencia previa del sujeto. Desde esta perspectiva, la calidad de vida aparece como la intersección de las áreas objetivas y subjetivas, incapaz de ser evaluada a partir de uno solo de sus dos polos o focos.

Entre los diferentes elementos integrantes del concepto de calidad de vida sobresale el de salud. No sólo es el primero que emerge históricamente, sino que también es el más básico de todos. Su importancia para la calidad de vida es doble: por un lado, el nivel de salud le afecta directamente, de forma que gran parte de la varianza de las actividades diarias puede estar en relación con la salud. Por otro lado, repercute indirectamente en ella, al afectar el resto de los valores o elementos que están presentes en la calidad de vida, como son el trabajo, el ocio, la autonomía, las relaciones sociales, etc. Desde esta perspectiva, la calidad de vida específicamente durante el embarazo significa el esfuerzo para que el proceso de gestación, el trabajo de parto y la transición a la maternidad supongan la menor alteración posible en la autonomía real de estas mujeres y entrañe las menores variaciones en sus vidas habituales.

Actualmente se observa un interés en el estudio de la calidad de vida y el proceso de embarazo. De hecho, en una investigación llevada a cabo con 42 mujeres adolescentes embarazadas, se observaron puntuaciones globales inferiores en los indicadores de calidad de vida durante el embarazo, cuando se compararon con los promedios de la población general. Los resultados que se obtuvieron pusieron de manifiesto la necesidad de facilitar ayuda y consejo psicológico a estas mujeres durante el proceso de embarazo, con la intención de disminuir posibles complicaciones médicas y psicológicas (Drescher, Monga, Williams, Promecene-Cook \& Scneider, 2003). En otra investigación realizada con 97 mujeres que presentaron complicaciones durante el embarazo y que fueron informadas de la necesidad de llevar a cabo abortos terapéuticos, se observaron decrementos significativos en los indicadores de calidad de vida medidos antes del aborto. Un resultado interesante de esta investigación fue que la disminución de las puntuaciones en algunos indicadores de calidad de vida estuvo asociada al conocimiento, por parte de la pareja de la mujer, de la necesidad de llevar a cabo el aborto (Westhoff, Picardoc \& Morrowa, 2003). A pesar de estas evidencias, poco se conoce sobre la relación que puedan tener ciertas diferencias individuales con la calidad de vida percibida de las embarazadas, especialmente su estilo emocional, en concreto el uso de la rumiación y la inhibición emocional, como formas de manejar las emociones asociadas a la experiencia del embarazo.

En relación a la interacción existente entre los estados de salud-enfermedad y las disposiciones individuales, tradicionalmente se han planteado dos relaciones. Por un lado, una relación causal, donde determinados rasgos de personalidad son factores directamente causantes de enfermedad (diversas investigaciones han tratado de correlacionar rasgos de personalidad con enfermedades como el cáncer o las afecciones cardiovasculares). Desde esta perspectiva, la reacción psicológica y fisiológica (de naturaleza genética, heredada) de un individuo cuando está expuesto a una situación específica, puede generar estados de salud o enfermedad, según sea el caso. De este modo, la personalidad es entendida como un estímulo que elicita una determinada respuesta (p. ej. Friedman \& Booth-Kewley, 1987; Turner, 1994).

Por otro lado, diferentes enfoques plantean que la personalidad del individuo es más bien una variable moderadora de los efectos que tienen los factores causales de enfermedad. Esta última aproximación plantea que los rasgos o disposiciones de personalidad pueden facilitar o inhibir la 
influencia de los factores causales de enfermedad (ejemplos de estas disposiciones de personalidad pueden ser el locus de control o la autoestima). Es decir, este enfoque entiende la salud y la enfermedad, como estados mediados cognitivamente, que reflejan la relación entre la persona y el entorno, según ésta interprete que dicha relación excede o no sus recursos, poniendo o no en peligro su bienestar (Denney \& Frisch, 1981; Sanderman \& Ranchor, 1997; Roger, 1995; 1996; 2007).

En el campo de la Psicología de la Salud comúnmente se ha tratado de relacionar variables como neuroticismo, extraversión, psicoticismo, rumiación o inhibición emocional, con factores como el estrés, las enfermedades cardiovasculares o el cáncer (ver Guarino, 2004). Dichos estudios tienen el objeto de demostrar los efectos que la personalidad y el funcionamiento emocional tienen sobre la salud y el bienestar del individuo como factores moderadores, ayudando a disipar la creencia de que las enfermedades están causadas primordialmente por factores biológicos.

Bajo esta perspectiva, la literatura muestra unos pocos estudios realizados con mujeres embarazadas. Específicamente, Lobel, Yali, Zhu, DeVincent y Meyer (2002) estudiaron un grupo de embarazadas de alto riesgo, determinando que características como el optimismo disposicional estuvo asociado a menores puntajes de distrés emocional, al facilitar la adopción de mejores estrategias de afrontamiento (activas y centradas en el problema) e incrementar la percepción de control que estas mujeres tienen sobre su embarazo. Por su parte, Canals, Esparó y Fernández-Ballart (2002) mostraron cómo los niveles de ansiedad durante el embarazo están asociados significativamente a características de personalidad como el neuroticismo; a la vez que Engelhard, van den Hout y Kindt (2003), en un estudio de carácter longitudinal, encontraron que elevados niveles de neuroticismo al inicio del embarazo estuvieron asociados a síntomas de estrés post-traumático en aquellas mujeres que habían sufrido la pérdida de su embarazo. Sin embargo y tal como se señaló anteriormente con respecto a la calidad de vida, el impacto del estilo emocional sobre la salud de este grupo particular de mujeres no se ha establecido aún.

Específicamente, la rumiación y la inhibición emocional son dimensiones que conforman el constructo de estilo emocional propuesto hace varios años por Roger y sus colaboradores (Roger \& Najarian, 1989; Roger \& Nesshoever, 1987; Roger, Guarino \& Olason, 2000), inicialmente descritas como parte del constructo de control emocional. La rumiación mide la tendencia de los individuos a mantenerse preocupados por eventos molestos ocurridos en el pasado, así como por situaciones o eventos estresantes futuros (preocupación sobre eventos que pueden ocurrir). Por otro lado, la inhibición emocional se refiere al "embotellamiento" o inhibición en la expresión de las emociones experimentadas, así como a la dificultad de revelar las propias emociones y sentimientos y de buscar a otros para recibir información o ser escuchados, cuando se hace frente a experiencias demandantes.

Ambas dimensiones mostraron predecir deterioro en la salud en diversas poblaciones bajo estrés (ver p. ej. Greco \& Roger, 2001; Guarino, 2004; 2009; Olason, 2001; Roger, 1996; Roger \& Najarian, 1998), especialmente la dimensión de rumiación, considerándolas estos autores factores moderadores importantes en la relación entre estrés y enfermedad; sin embargo la revisión de la literatura arrojó un solo estudio que relacionara alguna de estas dimensiones en mujeres embarazadas en su estadio final. Específicamente, Nieland y Roger (1993) estudiaron el caso concreto de mujeres en proceso de parto y el requerimiento de analgésicos para mitigar el dolor, determinando que una vez que factores como la edad, la duración del parto y el número de partos previos fueron controlados estadísticamente, existía una relación significativa entre la necesidad de más analgésicos y características de personalidad como la rumiación y la hipocondría (subdimensión del neuroticismo).

Si bien los hallazgos descritos hasta ahora constituyen antecedentes importantes en el área, es necesario seguir explorando las complejas dimensiones de la calidad de vida y la salud percibida de las mujeres embarazadas, sobre todo en el contexto socio-cultural latinoamericano, tratando de determinar específicamente la relación que pudiera existir entre estas variables y características personales como la rumiación y la inhibición emocional durante el embarazo, para lo cual se presenta el actual estudio con una muestra de embarazadas venezolanas. 


\section{Método}

\section{Participantes}

La muestra estuvo constituida por 94 mujeres que asistieron a control de embarazo en diversos centros asistenciales públicos y privados del Estado Miranda y Dto. Capital de Caracas-Venezuela, las cuales cumplían las siguientes condiciones: no reportar problemas de salud físico-mentales al momento de inicio del embarazo, asistir regularmente a control con un médico obstetra y con un nivel de instrucción mínimo de secundaria (completa). La edad promedio de estas mujeres fue de 27,56 años (mínima 16 - máxima 43).

\section{Instrumentos}

Para el desarrollo de este estudio, se emplearon los siguientes instrumentos de auto-reporte (cuestionarios):

- Cuestionario de Estilo Emocional (CEE- versión traducida y adaptada por Guarino, 2010, en prensa). Esta escala contiene 39 items, con un rango de respuesta dicotómica ciertofalso; 21 reactivos para la medición de la dimensión de inhibición emocional (IE) y 18 para la medición de la rumiación $(\mathrm{R})$, respectivamente. Los índices de consistencia interna alfa de Cronbach fueron bastante aceptables para los dos factores, siendo $=0.85$ para inhibición emocional y 0.82 para rumiación, para una muestra de 419 participantes (ver Guarino, 2010).

- Inventario de Síntomas Físicos de Cohen-Hoberman (CHIPS, 1983). Versión adaptada y validada a la población venezolana por Guarino (2004). Este inventario mide la percepción del estado de salud de los individuos a través de un listado de síntomas físicos. Consta de 33 ítems, a los que los participantes deben indicar la frecuencia en la que esos síntomas se han presentado en un período de cinco semanas. La versión hispana ha mostrado un adecuado nivel de consistencia interna ( $\alpha=0.88 ; \mathrm{N}=401)$ (ver Guarino, 2004; 2005).

- Cuestionario de Salud General de Golberg (GHQ - General Health Questionnaire). Versión hispana de Molina y Andrade (2002). Esta escala está compuesta por 28 reactivos que describen síntomas de ansiedad, depresión, somatización y disfunción social, respecto a los cuales los participantes deben reportar la frecuencia relativa con la que los han padecido en las últimas semanas, con un rango de posibilidades desde 1: No, en lo absoluto, hasta 4: Mucho más que lo habitual (Goldberg, 1972; Molina y Andrade, 2002). Las puntuaciones más altas reflejan una mejor salud. Los índices de consistencia interna (Alfa de Cronbach) resultaron óptimos para todas las dimensiones del GHQ en una muestra de 328 venezolanos, oscilando entre $\alpha=0,80$ y $\alpha=0,93$, con un $\alpha=0,93$ para la escala completa (Sojo, 2006).

- Cuestionario sobre Calidad de Vida: Satisfacción y Placer (Quality of life, enjoyment and satisfaction questionnaire, Q-LES-Q) (Bobes, Paz, Bascarán, Saiz y Bousoño, 2004). Este cuestionario de 93 items mide la calidad de vida percibida de los individuos a través de una exploración del grado de satisfacción con las múltiples actividades que realiza en su vida como: estado de salud física/actividades, estado de ánimo, trabajo, desarrollo de tareas de la casa, desarrollo de actividades académicas/estudio, actividades de tiempo libre, relaciones sociales y nivel de satisfacción general, a ser respondidas en una escala likert de 5 puntos desde Nunca hasta Muy a menudo o siempre. Las participantes del estudio debían responder cómo se habían sentido en cada una de las anteriores dimensiones durante la semana inmediatamente anterior.

\section{Procedimiento}

Tres auxiliares de investigación fueron entrenadas por la autora para administrar el cuadernillo contentivo de los instrumentos de auto-reporte a las mujeres embarazadas, las cuales participaron en el estudio en forma voluntaria, una vez expuestos los objetivos del mismo. Las participantes firmaron una carta de consentimiento presentada en la segunda página del cuadernillo en señal de 
conformidad con su participación en el estudio. Inicialmente, se hizo el contacto con un grupo de médicos gineco-obstetras que atendían consultas de control de embarazo en diferentes centros de salud públicos y privados de la ciudad capital (Caracas), para que permitieran el acercamiento de las investigadoras a sus pacientes durante las horas de espera a sus respectivas citas de control. El cuadernillo era respondido en las mismas salas de espera de los centros de salud, una vez obtenido el consentimiento de las participantes y determinado el cumplimiento de los criterios de inclusión en el estudio (ver características de la muestra).

\section{Resultados}

Los datos fueron analizados inicialmente a través de estadísticos descriptivos para establecer su tendencia central y dispersión, para posteriormente analizar las asociaciones entre las variables a través del método de correlación $r$ de Pearson.

\section{Análisis Descriptivo}

Tal como se observa en la tabla 1, los puntajes obtenidos por las participantes en las sub-escalas de rumiación e inhibición emocional son considerablemente bajos, ni siquiera alcanzando el valor medio posible de puntuación en los cuestionarios. Situación similar se observa con los puntajes de salud, indicando esto que las participantes se perciben a sí mismas con un adecuado estatus de salud. Con respecto a los indicadores de calidad de vida, los resultados en general señalan una tendencia hacia una calidad de vida percibida moderadamente buena, basados en el rango máximo posible de puntuación, aunque no suficientemente alta como para considerar que este grupo total de embarazadas esté disfrutando de una plena satisfacción de vida durante su proceso de gestación. Los datos de dispersión sugieren la presencia de puntuaciones extremas para estas sub-escalas. En consecuencia, es necesario investigar qué factores estarán asociados a las puntuaciones bajas obtenidas por algunas participantes y precisamente la próxima sección reportará los niveles de correlación entre estos indicadores y las dimensiones de estilo emocional estudiadas.

Tabla 1: Descriptivos de las variables estudiadas

Estilo Emocional

Rumiación

Inhibición Emocional

Indicadores de Salud

CHIPS

19.38

Somatización

Disfunción Social

Depresión

Calidad de vida

QLESQ Salud

QLES Estado ánimo

QLESQ Trabajo
0-132

0-112

$7-28$

$7-28$

$7-28$

$7-28$

$0-52$ 


\begin{tabular}{lccc} 
QLESQ Casa & 30.76 & 6.13 & $0-40$ \\
QLESQ Estudio & 28.42 & 8.76 & $0-40$ \\
QLESQ Tiempo libre & 15.26 & 6.34 & $0-24$ \\
QLESQ Relaciones sociales & 32 & 8.42 & $0-44$ \\
QLESQ Satisfacción General & 48.39 & 11.42 & $0-64$ \\
\hline
\end{tabular}

QLESQ $=$ Quality of Life: Enjoyment and satisfaction questionnaire; CHIPS= Cohen-Hoberman Inventory of Physical Symptoms; GHQ= General Health Questionnaire;

\section{Análisis de Correlación}

La tabla 2 muestra los resultados de las correlaciones entre las dimensiones de estilo emocional: rumiación e inhibición emocional y los indicadores de salud. Tal como se esperaba, las embarazadas que tienen más tendencia a usar la rumiación reportan significativamente más presencia de síntomas físicos, así como puntajes más altos en somatización, angustia y ansiedad y depresión. Finalmente, este grupo de mujeres reportan mayores niveles de deterioro general, en relación a sus contrapartes, que usan significativamente menos rumiación como estilo de reactividad emocional.

Tabla 2: Correlaciones entre Estilo Emocional e indicadores de salud

\begin{tabular}{lcccccc}
\hline & CHIPS & $\begin{array}{c}\text { Somatiza- } \\
\text { ción }\end{array}$ & $\begin{array}{c}\text { Angustia y } \\
\text { Ansiedad }\end{array}$ & $\begin{array}{c}\text { Disfunción } \\
\text { Social }\end{array}$ & Depresión & $\begin{array}{c}\text { GHQ } \\
\text { Total }\end{array}$ \\
\hline & & & & & & \\
Rumiación & $\mathbf{. 5 1 4 * *}$ & $\mathbf{- . 4 1 5 * *}$ & $\mathbf{- . 6 2 * *}$ & -.19 & $\mathbf{- . 5 1 * *}$ & $\mathbf{- . 5 4 * *}$ \\
& & & & & & \\
$\begin{array}{l}\text { Inhibición Emo- } \\
\text { cional }\end{array}$ & $\mathbf{. 2 4 *}$ & $\mathbf{- . 2 3 *}$ & $\mathbf{- . 2 6 *}$ & -.21 & $\mathbf{- . 4 1 * *}$ & $\mathbf{- . 3 5 * *}$ \\
\end{tabular}

CHIPS $=$ Cohen-Hoberman Inventory of Physical Symptoms; GHQ= General Health Questionnaire

Con respecto a la inhibición emocional, los resultados muestran un patrón muy similar a los anteriores, indicando que las embarazadas que tienen más dificultad para expresar sus emociones y en consecuencia tienden a inhibirlas, presentan simultáneamente mayor sintomatología física, así como mayor somatización y mayores niveles de angustia, ansiedad y depresión. Aunque la magnitud de las correlaciones no es tan elevada como en el caso de la correlación de estos indicadores con la rumiación, los resultados siguen siendo significativos.

Los resultados para los indicadores de calidad de vida son igualmente interesantes (ver tabla 3 ). Así, las mujeres embarazadas que tienen mayor tendencia a la rumiación perciben peor calidad de vida con respecto a su salud, estado de ánimo, trabajo, tiempo libre, relaciones sociales y en general menor satisfacción. Resultados similares se muestran para la inhibición emocional, de tal forma que las embarazadas que inhiben más sus emociones, reportan peor calidad de vida en relación a su estado de ánimo, trabajo, tiempo libre, relaciones sociales y satisfacción general, respectivamente. 
Tabla3: Correlaciones entre Estilo Emocional y calidad de vida

\begin{tabular}{|c|c|c|c|c|c|c|c|c|}
\hline & $\begin{array}{c}\text { QLESQ } \\
\text { Salud }\end{array}$ & $\begin{array}{l}\text { QLES } \\
\text { Estado } \\
\text { ánimo }\end{array}$ & $\begin{array}{l}\text { QLESQ } \\
\text { Trabajo }\end{array}$ & $\begin{array}{c}\text { QLESQ } \\
\text { Casa }\end{array}$ & $\begin{array}{l}\text { QLESQ } \\
\text { Estudio }\end{array}$ & $\begin{array}{c}\text { QLESQ } \\
\text { Tiempo } \\
\text { libre }\end{array}$ & $\begin{array}{c}\text { QLESQ } \\
\text { Rela- } \\
\text { ciones } \\
\text { sociales }\end{array}$ & $\begin{array}{l}\text { QLESQ } \\
\text { Satis- } \\
\text { facción } \\
\text { General }\end{array}$ \\
\hline Rumiación & $-.26 *$ & $-.58 * *$ & $-.29 *$ & -.19 & -.34 & $-.21 *$ & $-.37 * *$ & $-.37 * *$ \\
\hline $\begin{array}{l}\text { Inhibición } \\
\text { Emocional }\end{array}$ & -.20 & $-.41 * *$ & $-.29 *$ & -.08 & -.09 & $-.39 * *$ & $-.49 * *$ & $-.51 * *$ \\
\hline
\end{tabular}

QLESQ= Quality of Life: Enjoyment and satisfaction questionnaire

\section{Discusión}

El presente estudio de carácter descriptivo-correlacional se adscribe a una de las líneas de investigación desarrollada por la primera autora y sus colaboradores, la cual intenta determinar la relación entre diversas características personales con la manifestación del estrés y su consecuente impacto sobre la salud y la calidad de vida de los individuos, con el fin último de establecer las variables moderadoras del proceso (ver p. ej. Bethelmy y Guarino 2008a; 2008b; Guarino, 1998; 2004; 2005; Lousinha, 2009; Sojo y Guarino, 2010, en prensa). Específicamente para este estudio los autores se propusieron determinar la relación entre las dimensiones del constructo de estilo emocional - rumiación e inhibición emocional - descritas por Roger y sus colaboradores (Roger \& Najarian, 1989; Roger \& Nesshoever, 1987; Roger, Guarino \& Olason, 2000) y la salud y calidad de vida percibidas en mujeres embarazadas, grupo poco estudiado en nuestro contexto latinoamericano.

Tal como se esperaba, la rumiación y la inhibición emocional, como formas particulares de manejar y manifestar las emociones frente a las circunstancias cambiantes del entorno, resultaron ser disfuncionales para las mujeres gestantes, en función de su asociación significativa con una mayor presencia de síntomas físicos y psicológicos, así como por su relación con una peor calidad de vida percibida. Particularmente, la rumiación mostró tener un impacto mayor sobre la salud física y mental de las embarazadas que la inhibición emocional, tal como se había observado en estudios previos con otras muestras de participantes, usando el mismo cuestionario de estilo emocional en su versión anglosajona e indicadores de salud (Forbes \& Roger, 1999; Greco \& Roger, 2001; Guarino, 2004; Nieland \& Roger, 1993; Roger, 1996; Roger \& Najarian, 1998). La versión hispana del cuestionario (CEE) usada en el presente estudio, previamente había demostrado su valor predictivo, fundamentalmente en el caso de la rumiación, al explicar significativamente el deterioro físico y mental de una muestra de estudiantes venezolanos en período de adaptación a la universidad (Guarino, 2010, en prensa).

La rumiación, descrita como la tendencia de los individuos a mantenerse preocupados por eventos molestos ocurridos en el pasado o como serie de auto-afirmaciones focalizadas en los propios aspectos negativos, viene siendo investigada en el campo de la psicología clínica y de la salud como una característica individual altamente asociada a la depresión y a la ansiedad (p. ej. Nolen-Hoeksema, 2000; Nolen-Hoeksema \& Morrow, 1991; Nolen-Hoeksema, Parker y Larson, 1994; Rimes \& Watkins, 2005; Muris, Roelofs, Rassin, Franken \& Mayer, 2005; Watkins \& Moulds, 2005). Más aún, altos puntajes en la escala de rumiación han correlacionado significativamente con índices de recuperación fisiológica prolongada en individuos expuestos a estímulos estresores, tanto de laboratorio como diarios, lo cual sugiere un mantenimiento de tal activación aún después de que desaparecen tales estímulos (Roger \& Jamieson, 1987; Roger, 1988). Igualmente, la rumiación 
predijo elevados niveles de cortisol en orina en un grupo de enfermeras sometidas a un evento estresante natural (Roger \& Najarian, 1998). De acuerdo con Roger, la rumiación facilita la activación neurofisiológica asociada a la respuesta de estrés, por la vía de la activación sostenida del eje hipotalámico-hipofisiario- adrenal, comprometiendo la salud de los individuos en función de la liberación aumentada de cortisol y catecolaminas, con la consecuente depresión del sistema inmune (Roger, 2007; Roger \& Najarian, 1998).

No menos importante es el caso de la inhibición emocional, que representa el "embotellamiento" de las propias emociones y, en consecuencia, la dificultad en su expresión, cuyos efectos adversos sobre la salud también se han reportado en la literatura. Por ejemplo, Kaiser, Hinton, Krohne, Stewart y Burton (1995) mostraron que individuos con elevada inhibición emocional experimentan una recuperación lenta y demorada en su tensión muscular, luego de haber sido sometidos a estresores de laboratorio. Por otro lado, el "miedo a la revelación", que es una sub-escala altamente relacionada con la inhibición emocional y que describe la dificultad para abrirse a otras personas y comentar sobre los propios sentimientos y emociones, ha mostrado estar correlacionada positivamente con deterioro en el estatus de salud de estudiantes en períodos de estrés y adaptación (Forbes y Roger, 1999), así como con el uso de formas de afrontamiento disfuncionales en estudiantes universitarios y deterioro de la salud física y mental en un grupo de desempleados venezolanos(ver Guarino y Sojo, 2009). En el presente estudio se demostró el impacto negativo de este estilo de respuesta emocional, al asociarse a una mayor presencia de síntomas físicos y psicológicos en las mujeres gestantes durante su primer trimestre, pero fundamentalmente su efecto nocivo parece ser mayor sobre la calidad de vida percibida de estas mujeres, indicador que no había sido estudiado anteriormente en su relación con el estilo emocional. Tal resultado indica que la experiencia del embarazo podría verse mucho más enriquecida y ser más placentera si las mujeres fueran capaces de expresar más abiertamente sus emociones, malestares, sentimientos y/o preocupaciones relacionadas con las condiciones y características de este proceso.

En síntesis, este estudio revela que los estilos de respuesta emocional como la rumiación y la inhibición emocional pueden resultar muy dasadaptativos para la salud y el bienestar global de las mujeres embarazadas en el inicio de su período de gestación, condición que compromete no sólo su integridad, sino la del ser que se encuentra en formación. Estos datos deberían llamar la atención sobre la importancia de asumir políticas de prevención y promoción de salud para esta población particular, a través de la asesoría y el apoyo de profesionales de la psicología que intervinieran sobre el control y modificación de tales características personales, reduciendo así el riesgo de consecuencias adversas sobre la salud y bienestar de la mujer embarazada, fomentando una mejor calidad de vida en tan importante ciclo vital. Concretamente, no basta sólo con proporcionarles a este grupo de mujeres atención médica en los aspectos orgánicos y fisiológicos del embarazo, sino que debería complementarse tal atención con la incorporación de psicólogos que registraran sus cambios emocionales y conductuales asociados, con el objeto de promover un embarazo integralmente saludable.

\section{Referencias}

Bethelmy, L., y Guarino, L. (2008). Estrés laboral, afrontamiento, sensibilidad emocional y síntomas físicos y psicológicos en médicos venezolanos. Revista Colombiana de Psicologia, 17, 43-58.

Bethelmy, L., y Guarino, L. (2008b). Afrontamiento y sensibilidad emocional como moderadores de la relación estrés-salud en médicos venezolanos. Summa Psicológica UST, 5, 3-16.

Bobes, J., Paz, M. Bascarán, M.T., Sáiz, P., y Bousoño, M. (2004). Cuestionario sobre Calidad de vida: Satisfacción y Placer (Quality of life, enjoyment and satisfaction questionnaire, Q-LES-Q). Banco de Instrumentos básicos para la práctica de la psiquiatría clínica. 3ra. Edición. Psiquiatría Editores, S.L. Barcelona. 
Canals, J., Esparó, G., \& Fernández-Ballart, J. (2002). How anxiety levels during pregnancy are linked to personality dimensions and sociodemographic factors. Personality and Individual Differences, 33, 253-259.

Denney, D. R., \& Frisch, M.B. (1981). The role of neuroticism in relation to life stress and illness. Journal of Psychosomatic Research, 25, 303-307.

Drescher, K.; Monga, M.; Williams, P.; Promecene-Cook, P. \& Scneider, K. (2003). Perceived quality of life in pregnant adolescent girls. American Journal of Obstetritia Gynecology, 188 (5): 1231-1233.

Engelhard, I., van den Hout, M., \& Kindt, M. (2003). The relationship between neuroticism, pretraumatic stress, and post-traumatic stress: a prospective study. Personality and Individual differences, 35, 381-388.

Forbes, A. y Roger, D. (1999). Stress, social support and fear of disclosure. British Journal of Health Psychology, 4, 165-179.

Friedman, H., \& Booth-Kewley, S. (1987). The “disease-prone personality”. A Meta-Analitic View of the Construct. American Psychologist, 42, 6, 539-555.

Greco, V y Roger, D. (2001). Coping with uncertainty: the construction and validation of a new measure. Personality and Individual Differences, 31, 519-534.

Guarino, L. (1998). Variables asociadas al estrés académico y a la salud mental en estudiantes de la USB. Manuscrito sin publicar. Universidad Simón Bolívar, Caracas-Venezuela.

Guarino, L. (2004). Emotional Sensitivity: a new measure of emotional lability and its moderating role in the stress-illness relationship. Tesis Doctoral sin publicar, Universidad de York, Inglaterra.

Guarino, L. (2005). Sensibilidad Emocional: Su Rol Moderador en la Relación Estrés-Enfermedad. Psicología Conductual, 13 (2), 217-230.

Guarino, L. (2009). Validación del Modelo de Sensibilidad Emocional. Propuesta de un Modelo de Mediación Moderada. Manuscrito sin publicar (Trabajo de Ascenso a profesor Titular). Universidad Simón Bolívar. Caracas.

Guarino, L. (2010, en prensa). Adaptación y Validación de la versión hispana del Cuestionario de Estilo Emocional. Universitas Psychologica.

Guarino, L. y Sojo, V. (2009). Adaptación y validación del ITQ (Interpersonal Trust Questionnaire). Una nueva medida del Apoyo Social. Avances en Psicología Latinoamericana, 27, 192-206.

Kaiser, J., Hinton, J., Krohne, H., Stewart, R., \& Burton, R. (1995). Coping dispositions and physiological recovery from a speech preparation stressor. Personality and Individual Differences, $19,1-11$.

Levi, L. y Anderson, L. (1980). La tensión psicosocial. Población, ambiente y calidad de vida. México D.F.: El Manual Moderno.

Lobel, M., Yali, M.A., Zhu, W., DeVincent, C., \& Meyer, B. (2002). Beneficial associations between optimistic disposition and emotional distress in high-risk pregnancy. Psychology and health, 17, $1,77-95$.

Lousinha, A. (2009). Diferencias individuales como moderadoras de la relación estrés-salud en docentes universitarios. Manuscrito sin publicar, Universidad Simón Bolívar, Caracas, Venezuela.

Molina y Andrade (2002). La estructura factorial del GHQ-60 en una muestra de población general: una versión escalar para población española. Revista Internacional On-line, 1, 2. Recuperado el 20 de Abril de 2008 de la pagina web: http://www.psiquiatria.com/articulos/psiqsocial/8633/ 
Muris, P., Roelofs, J., Rassin, E., Franken, I., y Mayer, B. (2005). Mediating effects of rumination and worry on the links between neutoticism, anxiety and depression. Personality and Individual Differences, 39, 1105-1111.

Nieland, M., \& Roger, D. (1993). Emotion control and analgesia in labour. Personality and Individual Differences, 14, 6, 841-843.

Nolen-Hoeksema, S. \& Morrow, J. (1991). A prospective study of depression and posttraumatic stress symptoms after a natural disaster: the Loma Pietra earthquake. Journal of Personality and Social Psychology, 61, 115-121.

Nolen-Hoeksema, S. (2000). The role of rumination in depressive disorders and mixed anxiety/ depressive symptoms. Journal of Abnormal Psychology, 109, 504-511.

Nolen-Hoeksema, S., Parker, L. \& Larson, J. (1994). Ruminiative coping with depressed mood following loss. Journal of Personality and Social Psychology, 67, 92-104.

Olason, D. (2001). Optimism, Pessimism and "Fighting spirit": Their role in moderating the stress response. Tesis Doctoral sin publicar, Universidad de York - Inglaterra.

Rimes, K. \& Watkins, E. (2005). The effects of self-focused rumination on global negative selfjudgements in depression. Behavior Research and Therapy, 43, 1673-1681.

Roger, D. (1988, Julio). The role of emotion control in human stress responses. Comunicación presentada en la Conferencia Anual de la Sociedad Británica de Psicología, Universidad de Leeds, Inglaterra.

Roger, D. (1995, Abril). The mechanics of stress: a model for the relationship between stress, health and personality. Comunicación presentada en la 5ta. Conferencia Internacional en Manejo del Estrés, Noordwijkerhout, Holanda.

Roger, D. (1996, Julio). The role of cognitive rumination, coping styles and self-esteem in moderating adaptational responses to stress. Comunicación presentada en la 8va Conferencia Europea de Personalidad, Universidad de Ghent.

Roger, D. (2007). Self-esteem, stress, and emotion. En G. Fink (Ed.), Encyclopedia of Stress (2nd Ed.). Oxford: Academic Press.

Roger, D., Guarino, L., y Olason, D. (2000, Agosto). Emotional style and health: A new three-factor model. Comunicación presentada en la 14va. Conferencia Europea de Psicología de la Salud, Leiden, Holanda.

Roger, D., y Najarian, B. (1989). The construction and validation of a new scale for measuring emotional control. Personality and Individual Differences, 10, 845-853.

Roger, D., y Najarian, B. (1998). The relationship between emotional rumination and cortisol secretion under stress. Personality and Individual Differences, 24, 4, 531-538.

Roger, D., y Nessshoever, W. (1987). The construction and preliminary validation of a scale for measuring emotional control. Personality and Individual Differences, 8, 527-534.

Roger, D., y Jamieson, J. (1988). Individual Differences in delayed heart-rate recovery following stress: The role of extraversion, neuroticism and emotional control. Personality and Individual Differences, 9, 4, 721-726.

Sanderman, R., \& Ranchor, A. V. (1997). The predictor status of personality variables: Etiological significance and their role in the course of disease. European Journal of Personality, 11, 359382.

Shin, D.C. \& Johnson, D.M. (1978). Avowed happiness as and overall assessment of the quality of life. Social Indicators Research, 5, 475-492. 
Sojo, V. y Guarino, L. (2010, en prensa). Manejo emocional como moderador de la relación estréssalud en desempleados venezolanos. Psicología Conductual.

Turner, R. J. (1994). Cardiovascular reactivity and stress: Patterns of physiological response. New York: Plenum P.

Watkins, E., \& Moulds, M. (2005). Positive beliefs about rumination in depression - a replication and extension. Personality and Individual Differences, 39, 73-82.

Westhoff, C.; Picardoc, L. \& Morrowa, E. (2003). Quality of life following early medical or surgical abortion. Contraception, 67, 41-47. 
